\title{
ESR-Untersuchungen zur Radikalbildung in röntgenbestrahlten zinnorganischen Verbindungen
}

\author{
K. Höppner und G. Lassmann \\ Institut für Angewandte Isotopenforschung und Institut für Biophysik der DAW zu Berlin *
}

(Z. Naturforsch. 23 a, 1758-1762 [1968] ; eingegangen am 10. August 1968)

\begin{abstract}
Formation of radicals in tin-organic compounds of the type $\mathrm{R}_{4} \mathrm{Sn}\left(\mathrm{R}: \mathrm{CH}_{3}, \mathrm{C}_{2} \mathrm{H}_{5}, \mathrm{C}_{2} \mathrm{H}_{6}, \mathrm{Cl}, \mathrm{Br}\right)$ as well as $\left(\mathrm{CH}_{3}\right)_{3} \mathrm{SnCl}$ and $\left(\mathrm{CH}_{3}\right)_{3} \mathrm{Sn}-\mathrm{Sn}\left(\mathrm{CH}_{3}\right)_{3}$ irradiated with $\mathrm{X}$-rays at $77^{\circ} \mathrm{K}$ has been investigated by ESR. The change of spectra after stepwise annealing of the sample shows that a least two types of radicals are present. In some cases trivalent tin radicals $\mathrm{R}^{\prime}\left(\mathrm{CH}_{3}\right)_{2} \mathrm{Sn}\left(\mathrm{R}^{\prime}: \mathrm{CH}_{3}\right.$ or $\left.\mathrm{Sn}\left(\mathrm{CH}_{3}\right)_{3}\right)$ with an unpaired electron localized at the $\mathrm{Sn}$ atom with $g=2.02$ have been detected. A further radical of the type $\equiv \mathrm{Sn}-\dot{\mathrm{C}} \mathrm{H}_{2}$ with a tin hyperfine structure of 132 and $160 \mathrm{Gau}$ resp. has been identified by the hyperfine structure of the magnetic ${ }^{117} \mathrm{Sn}$ - and ${ }^{119} \mathrm{Sn}$-nuclei. A mesomeric stabilized radical in $\left(\mathrm{C}_{6} \mathrm{H}_{5}\right)_{4} \mathrm{Sn}$ is unusually stable, as the ESR spectrum did not change from $77^{\circ} \mathrm{K}$ till $423^{\circ} \mathrm{K}$. The yield of radicals in $\left(\mathrm{C}_{6} \mathrm{H}_{5}\right){ }_{4} \mathrm{Sn}$ is smaller by two orders of magnitude than in aliphatic tin-organic compounds.
\end{abstract}

Bei Untersuchungen der Photolyse und Radiolyse aliphatischer zinnorganischer Verbindungen wurden von verschiedenen Autoren ${ }^{1,2}$ Reaktionsmechanismen vorgeschlagen, die das Auftreten von Organozinn-Radikalen als Intermediärprodukte postulieren. Zum Nachweis solcher kurzlebigen paramagnetischen Zentren haben wir die ESR-Spektroskopie benutzt. Frühere Versuche an tritiummarkiertem Dimethyldiäthylzinn ergaben Hinweise auf das Vorhandensein von Organozinn-Radikalen ${ }^{3}$. Von Sснмidt et al. ${ }^{4}$ wurden bei $77^{\circ} \mathrm{K}$ ESR-Signale an UV-bestrahltem Triphenylstannan und Triisobutylstannan nachgewiesen, die den in ${ }^{3}$ erhaltenen sehr ähnlich sind und ebenfalls als dreibindige Zinnradikale interpretiert wurden. Schließlich konnte auf chemischem Wege durch Hydrierung in benzolischer Lösung ein Radikal erzeugt werden, das als „mesomeres Zinnradikal" gedeutet wurde ${ }^{5}$.

In der vorliegenden Arbeit wird über Ergebnisse zur Radikalbildung bei der Radiolyse einfacher Organozinn-Verbindungen mit Hilfe der ESR berichtet.

\section{Experimentelles}

Folgende Substanzen wurden untersucht:

$$
\begin{array}{ll}
\text { Tetramethylzinn } & \left(\mathrm{CH}_{3}\right)_{4} \mathrm{Sn} \\
\text { Trimethylzinnchlorid } & \left(\mathrm{CH}_{3}\right)_{3} \mathrm{SnCl}
\end{array}
$$

* X 1115 Berlin-Buch, Lindenberger Weg 70.

1 G. A. Razunaev, N. S. Vyazankin u. O. A. Shchepetkova, Tetrahedron 18, 667 [1962].

${ }^{2}$ K. Höppner, Proc. 2nd Tihany Sympos. Radiation Chem. 1966, p. 33, Budapest 1967.

3 W. Damerau, G. Lassmann u. H. G. Thom, Z. Phys. Chem. Leipzig 223, 99 [1963].

$\begin{array}{ll}\text { Tetraphenylzinn } & \left(\mathrm{C}_{6} \mathrm{H}_{5}\right)_{4} \mathrm{Sn} \\ \text { Tetraäthylzinn } & \left(\mathrm{C}_{2} \mathrm{H}_{5}\right)_{4} \mathrm{Sn} \\ \text { Hexamethyldizinn } & \left(\mathrm{CH}_{3}\right)_{3} \mathrm{SnSn}\left(\mathrm{CH}_{3}\right)_{3} \\ \text { Zinntetrachlorid } & \mathrm{SnCl}_{4} \\ \text { Zinntetrabromid } & \mathrm{SnBr}_{4}\end{array}$

Tetramethylzinn und Tetraäthylzinn wurden durch Grignardierung von Zinntetrachlorid hergestellt und durch präparative Gaschromatographie gereinigt.

Die Darstellung von Trimethylzinnchlorid erfolgte durch Komproportionierung von Tetramethylzinn und Zinntetrachlorid; es wurde durch Destillation und Sublimation im Hochvakuum gereinigt.

Tetraphenylzinn erhielten wir freundlicherweise vom VEB Elektrochemisches Kombinat Bitterfeld und verwendeten dies ohne weitere Reinigung.

Hexamethyldizinn wurde aus Trimethylzinnbromid und Natrium in flüssigem Ammoniak erhalten und durch präparative Gaschromatographie gereinigt.

Handelsübliches Zinntetrachlorid wurde im Vakuum destilliert; Zinntetrabromid aus metallischem Zinn und Brom dargestellt und im Hochvakuum destilliert.

Da diese Verbindungen - außer Tetraphenylzinn bei Zimmertemperatur Flüssigkeiten sind oder wenig oberhalb Zimmertemperatur schmelzen, mußten alle Röntgen-Bestrahlungen und ESR-Messungen bei $77^{\circ} \mathrm{K}$ durchgeführt werden. Der niedrige Schmelzpunkt, z. B. von Tetraäthylzinn, erforderte spezielle Präparationen und komplizierte Manipulationen. Die Bestrahlung erfolgte in einem Tieftemperatur-Kryostaten ${ }^{6}$ mit 1,0 MeV-Röntgen-Strahlen am Kaskaden-Generator des Institutes für Biophysik. Die Dosisleistung betrug dabei maximal $10^{6} \mathrm{rad} / \mathrm{min}$, so daß Gesamtbestrahlungsdosen

4 U. Schmidt, K. G. Kabitzke, K. Markau U. W. P. Neumann, Chem. Ber. 98, 3827 [1965].

5 H. B. Stegmann u. K. Scheffler, Tetrahedron Letters 1964 (46), 3387.

${ }^{6}$ G. Lassmann, Exp. Techn. Phys. 11, 373 [1963]. 
von $10^{8} \mathrm{rad}$ auch bei $77^{\circ} \mathrm{K}$ ohne weiteres zur Verfügung standen.

Die Substanzen wurden nach mehreren EinfrierAuftau-Entgasungsschritten mit flüssigem Stickstoff in dünnwandige Glasröhrchen einkondensiert und diese im Hochvakuum abgeschmolzen.

Die Verbindungen sind bei $77^{\circ} \mathrm{K}$ glasig erstarrt und damit nicht umschüttbar. Zur Beseitigung des störenden Signals vom bestrahlten Glas mußten also von der üblichen Technik abweichende Wege beschritten werden.

Die Küvette mit der bestrahlten Probe wurde unter flüssigem Stickstoff geöffnet und das bestrahlte Material ebenfalls unter flüssigem Stickstoff rasch in ein vorgekühltes unbestrahltes Glasröhrchen überführt, welches dann verkittet wurde. In einigen Fällen gelang es, durch Eintropfen der Substanz in flüssigen Stickstoff Kügelchen zu erzeugen, die dann in der bestrahlten Küvette nach Ausheizen des leeren Endes und erneuter Abkühlung in dieses Ende umgeschüttet werden konnten.

Die ESR-Messung erfolgte in einem üblichen EinsatzKryostaten, in dem die Probe von flüssigem Stickstoff umgeben war. Die unvermeidlichen Siedeblasen wurden durch Glaswolle so klein gehalten, daß die dadurch bedingten Fluktuationen von der Frequenzstabilisierung weitgehend ausgeregelt werden konnten. Ein Vibrieren der Probe konnte durch Festklemmen des Röhrchens mit einem porösen Schaumstoff vermieden werden. Zur Erwärmung der Probe auf Temperaturen von $77^{\circ} \mathrm{K}$ bis Zimmertemperatur diente ein $\mathrm{N}_{2}$-Dampfkryostat.

Die Registrierung der ESR-Spektren erfolgte mit einem empfindlichen Superheterodyn-Spektrometer ${ }^{7}$ als 1. bzw. 2. Ableitung der Absorption. Zur Festlegung des Feldmaßstabes und des $g$-Faktors diente eine dünne Glaskapillare mit einer wäßrigen Lösung von Peroxylamindisulfonat, die neben dem Kryostaten im Resonator angebracht und deren Spektrum mitregistriert wurde.

\section{Ergebnisse und Diskussion}

\section{Tetramethylzinn}

Das in Abb. 1 gezeigte ESR-Spektrum von bei $77^{\circ} \mathrm{K}$ bestrahltem Tetramethylzinn hat eine Gesamtbreite von $170 \mathrm{Gauß}$ und ist stark asymmetrisch ${ }^{8}$. Es besteht aus 2 überlagerten Spektren: einem intensiven Triplett mit einem $g$-Faktor von 2,0067 und einer stark asymmetrischen breiten Linie mit $g=2,023$, welche einen Teil des Tripletts verdeckt.

Das Triplett wurde bereits in einer früheren Arbeit ${ }^{10}$ einem zinnorganischen Radikal der Struktur I

7 N. Klimes, G. Lassmann u. J. Schmidt, Exp. Techn. Phys., im Druck.

8 Dieses Spektrum wurde bereits früher ${ }^{9}$ aufgenommen, aber nicht hinreichend interpretiert.

9 W. Gordy u. C. G. McCormick, J. Am. Chem. Soc. 78, 3243 [1956].

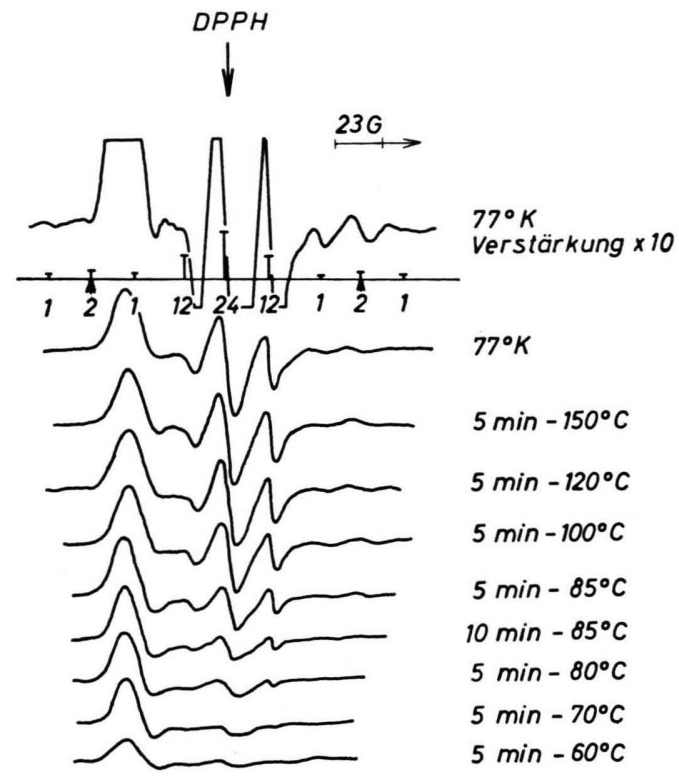

Abb. 1. ESR-Spektren von $\left(\mathrm{CH}_{3}\right)_{4} \mathrm{Sn}$ nach Röntgen-Bestrahlung bei $77^{\circ} \mathrm{K}$ und anschließender Erwärmung der Probe. ESR-Messung stets bei $77^{\circ} \mathrm{K}$. 1. Ableitung.

zugeordnet. Die beiderseits des Haupt-Tripletts erkennbaren schwachen Satellitenlinien resultieren demnach aus der Hyperfeinstruktur (Hfs) der beiden zu insgesamt 16,35\% auftretenden Zinnisotope mit dem Kernspin $I=1 / 2$ und unterstützen die vorgeschlagene Struktur I.
I:<smiles>[CH2][Sn](C)(C)C</smiles>

II:<smiles>C[Sn](C)(C)C</smiles>

$$
\begin{aligned}
& g \quad=2,0067 \\
& a_{\mathrm{H}}=21 \mathrm{G} \\
& a_{\mathrm{Sn}}=132 \mathrm{G} \\
& g=2,023 \\
& \text { asymmetrisch }
\end{aligned}
$$

Das unterschiedliche Temperaturverhalten der asymmetrischen etwa $15 \mathrm{Gau}$ breiten Linie bei $g=2,023$ (Abb. 1) im Vergleich zum 1:2 : 1-Triplett weist auf die Existenz eines weiteren Radikaltyps hin. Diese Linie tritt überdies auch im Spektrum des bestrahlten Hexamethyldizinns auf (Abb. 4).

Die bisher in der Literatur beschriebenen Zinnradikale ${ }^{4,11,12}$, bei denen sich das unpaarige Elek-

10 G. Lassmann u. K. Höppner, Z. Naturforsch. 23 a, 662 [1968].

11 R. L. Morehouse, J. J. Christiansen u. W. Gordy, J. Chem. Phys. 45, 1751 [1966].

12 M. Magat, H. Leray u. G. Rogen, Z. Vsesojus. Khim. Obschtsch. XI, 223 [1966]. 
tron unmittelbar am Zinnatom befindet, zeigen wegen stärkerer Spin-Bahn-Kopplung des 5p-Elektrons $g$-Faktoren, die vom Wert des freien Elektrons erheblich abweichen. So zeigen das $\dot{\mathrm{SnH}}_{3}$-Radikal bei $4,2^{\circ} \mathrm{K} g$-Werte von 2,003 bis $2,025^{11}$ und das $\left(\mathrm{i}-\mathrm{C}_{4} \mathrm{H}_{9}\right)_{3} \dot{\mathrm{S}}$-Radikal solche von 2,002 bis 2,034 (l. c. ${ }^{4}$ ).

Es liegt deshalb nahe, die Linie bei 2,023 einem Sn-Radikal, bei dem das unpaarige Elektron direkt am Zinnatom lokalisiert ist (Struktur II), zuzuordnen. Da es sowohl beim Tetramethylzinn als auch beim Hexamethyldizinn auftritt, wird ein $\left(\mathrm{CH}_{3}\right)_{3} \dot{\mathrm{S} n}$ Radikal angenommen, in welchem wegen der starken $g$-Anisotropie eine $\mathrm{CH}_{3}$-Protonen-Hfs im glasigen oder polykristallinen Zustand nicht aufgelöst ist (vgl. dazu auch ${ }^{11}$ ).

Die bei der Radiolyse des Tetramethylzinns bei Zimmertemperatur auftretenden Produkte $\left[\mathrm{H}_{2}\right.$, $\mathrm{CH}_{4}, \mathrm{C}_{2} \mathrm{H}_{6}, \quad\left(\mathrm{CH}_{3}\right)_{3} \mathrm{SnC}_{2} \mathrm{H}_{5}, \quad\left[\left(\mathrm{CH}_{3}\right)_{3} \mathrm{Sn}\right]_{2}$ und $\left(\mathrm{CH}_{3}\right)_{3} \mathrm{SnCH}_{2} \mathrm{Sn}\left(\mathrm{CH}_{3}\right)_{3}$ ] legen das Auftreten der beiden auch mit der ESR nachgewiesenen Radikale I und II nahe, wobei folgender Mechanismus angenommen wird:

$$
\begin{gathered}
\left(\mathrm{CH}_{3}\right)_{4} \mathrm{Sn} \sim\left(\mathrm{CH}_{3}\right)_{3} \mathrm{Sn}+{ }^{\circ} \mathrm{CH}_{3}, \\
\mathrm{CH}_{3}+\left(\mathrm{CH}_{3}\right)_{4} \mathrm{Sn} \rightarrow \mathrm{CH}_{4}+\left(\mathrm{CH}_{3}\right)_{3} \mathrm{SnCH}_{2} .
\end{gathered}
$$

Das Auftreten von Wasserstoff könnte auf die Abspaltung von Wasserstoffatomen zurückzuführen sein, die allerdings bei $77^{\circ} \mathrm{K}$ in diesem Gitter nicht stabilisierbar sind und deshalb im ESR-Spektrum fehlen:

$$
\left(\mathrm{CH}_{3}\right)_{4} \mathrm{Sn} \sim \rightarrow\left(\mathrm{CH}_{3}\right)_{3} \mathrm{SnC}_{2} \mathrm{H}_{2}+\mathrm{H} .
$$

Auch die Radiolyse von Neopentan ${ }^{13}$ liefert hauptsächlich ein durch Abtrennung einer Methylgruppe gebildetes t-Butylradikal. Daneben tritt in geringer Konzentration aber auch ein $\left(\mathrm{CH}_{3}\right)_{3} \mathrm{C}-\dot{\mathrm{C}} \mathrm{H}_{2}$-Radikal mit einem 1:2:1-Triplett und einer Aufspaltung von 22,7 Gauß auf, was auf gewisse Ähnlichkeiten im Verhalten der Kohlenstoff- und der Zinn-Verbindung gegenüber Radiolyse deutet.

Sowohl in ${ }^{5}$ als auch in ${ }^{10}$ wurden OrganozinnRadikale mit Zinn-Hfs durch die Satelliten der magnetischen Zinnisotope mit dem Kernspin 1/2 identifiziert. Um so überraschender erscheint die Tatsache, da $\beta$ in den bisher publizierten dreibindigen Zinn-Radikalen, bei denen das unpaarige Elektron direkt am Zinnatom lokalisiert ist ${ }^{\mathbf{4}} \mathbf{1 1 , 1 2}$, in

13 R. W. Fessenden u. R. H. Schuler, ESR Studies of Transient Alkyl Radicals, Mellon Institute, Pittsburgh 1963. keinem Fall derartige Zinn-Dubletts als schwache Linien oberhalb und unterhalb von $g=2$ beobachtet werden. Die Gründe für das Ausbleiben dieser Satelliten wurden in diesen Arbeiten nicht diskutiert. Eine mögliche Erklärung dafür wäre, daß bei dreibindigen Zinnradikalen die Hfs-Aufspaltung durch das Zentralatom sehr groß wird und weitab vom normalerweise überstrichenen Magnetfeldbereich liegt. Wir haben deshalb an bestrahltem Zinntetrachlorid und -bromid, von denen bekannt ist ${ }^{12}$, daß sie nach Radiolyse dreibindige Zinnradikale ergeben, das Spektrum vom Magnetfeld Null bis über $5000 \mathrm{Gau}$ aufgenommen. Die bei kleinerem Feld gelegene Dublett-Komponente müßte damit erfaßt werden, wenn sie überhaupt nachweisbar ist. Weder bei den Zinntetrahalogeniden noch bei den Tetraalkylstannanen und dem Hexaalkyldistannan konnte zwischen 0 und $5000 \mathrm{Gauß}$ außer bei $g=2$ eine weitere Resonanz nachgewiesen werden. Der Grund hierfür könnte darin liegen, daß die Hfs-Aufspaltung mehrere tausend Gauß groß ist, wodurch die Linienbreite in polykristallinen Proben sehr verwaschen wird und die ohnehin schon geringe Intensität der Satelliten deshalb unter die Nachweisgrenze fällt. Diese Annahme wird unterstützt durch eine Arbeit von Sugibuchi ${ }^{14}$, in der von $\mathrm{Sn}^{3+}$-Ionen in $\mathrm{ZnS} \mathrm{Hfs-}$ Aufspaltungen von im Mittel $5700 \mathrm{Gau}$ gefunden wurden. Diese Aufspaltung gilt zwar für ein reines 5 s-Elektron, durch Konfigurationswechselwirkung dürften aber auch beim dreibindigen Zinnradikal 5s-Anteile entstehen, die zu erheblichen Aufspaltungen führen, so daß Satellitenlinien von Zinnradikalen im festen Zustand nur in Einkristallen (wie in ${ }^{14}$ ) auflösbar sein dürften.

\section{Tetraäthylzinn}

Abb. 2 zeigt die 2.Ableitung des ESR-Spektrums vom Tetraäthylzinn (bestrahlt und gemessen bei $\left.77^{\circ} \mathrm{K}\right)$. Als Grundstruktur erkennt man ein Quintett mit dem annähernden Intensitätsverhältnis von $1: 4: 6: 4: 1$ und einer mittleren Aufspaltung von $22 \pm 2$ Gauß. Beim Tempern nehmen alle 5 Komponenten im gleichen Verhältnis ab. Außerdem scheint eine weitere Mehrlinienstruktur geringer Intensität überlagert zu sein. Satellitenlinien von einer ZinnHfs wie beim Tetramethylzinn waren nicht zu beobachten. Diese Befunde legen die Existenz eines Ra-

${ }^{14}$ K. Sugibuchi, Phys. Rev. 153, 404 [1967]. 


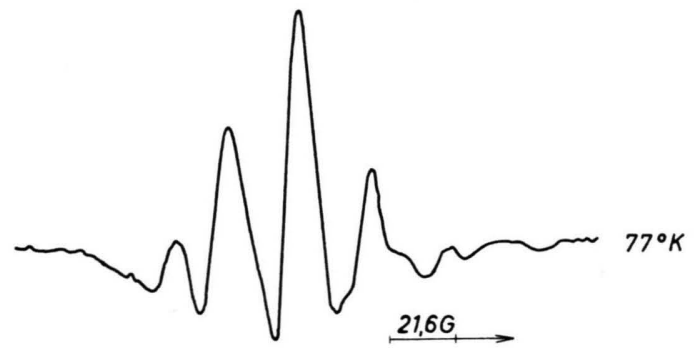

Abb. 2. ESR-Spektrum von $\left(\mathrm{C}_{2} \mathrm{H}_{5}\right)_{4} \mathrm{Sn}$ nach Röntgen-Bestrahlung bei $77^{\circ} \mathrm{K}$. 2. Ableitung.

dikals mit vier nahezu äquivalenten Protonen, die mit $22 \mathrm{Gauß}$ an der $\mathrm{Hfs}$ beteiligt sind, nahe. Eine Wechselwirkung mit dem Zinnkern ist nicht nachzuweisen. Als möglicher Radikaltyp wird deshalb folgende Struktur vorgeschlagen:<smiles>CCC(CC)(CC)[Si](CC)(CC)CC</smiles>

Aus den Radiolyseprodukten $\left(\mathrm{H}_{2}, \mathrm{CH}_{4}, \mathrm{C}_{2} \mathrm{H}_{6}\right.$, $\mathrm{C}_{2} \mathrm{H}_{4}, \mathrm{C}_{4} \mathrm{H}_{10},\left(\mathrm{C}_{2} \mathrm{H}_{5}\right){ }_{3} \mathrm{SnCH}_{3},\left(\mathrm{C}_{2} \mathrm{H}_{5}\right)_{3} \mathrm{SnC}_{4} \mathrm{H}_{9}$ und höhere Organozinn-Verbindungen) ist belegt, daß dieses Radikal tatsächlich auftreten muß und wahrscheinlich zum Teil durch Abspaltung eines Wasserstoffatoms vom Ausgangsmolekül gebildet wird.

\section{Trimethylzinnchlorid}

Das ESR-Spektrum des bestrahlten Trimethylzinnchlorid zeigt ein $1: 3: 3: 1$-Quartett mit einem $g$ Faktor von 2,004 und einer Aufspaltung von $22 \pm 1$

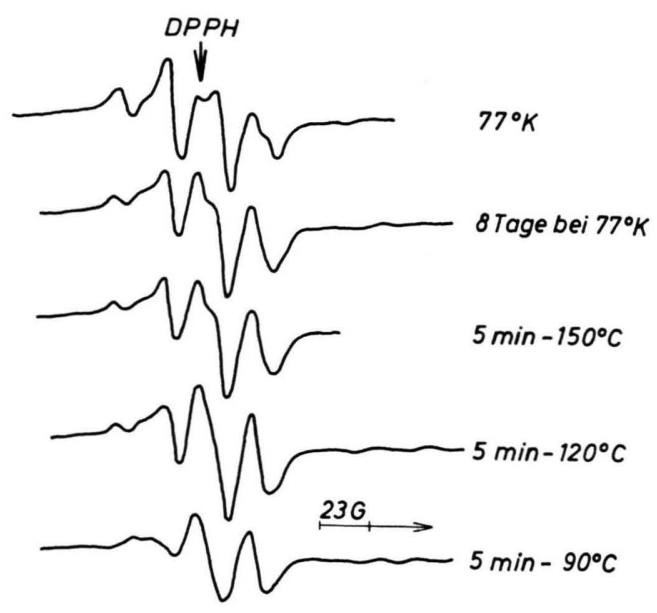

Abb. 3. ESR-Spektren von $\left(\mathrm{CH}_{3}\right)_{3} \mathrm{SnCl}$ nach Röntgen-Bestrahlung bei $77^{\circ} \mathrm{K}$ und anschließender Erwärmung der Probe (wie in Abb. 1).
Gauß (Abb. 3). Dieser für das Methylradikal typischen Struktur muß - wie die Erwärmungskurven in Abb. 3 zeigen - noch ein Spektrum eines weiteren Radikaltyps überlagert sein. Nach Erwärmung der Probe für 5 Minuten auf $-120{ }^{\circ} \mathrm{C}$ bzw. $-90^{\circ} \mathrm{C}$ ist das Methyl-Spektrum nahezu verschwunden und ein $1: 2$ : 1-Triplett mit $g=2,009$ und einer Aufspaltung von $20 \pm 1 \mathrm{Gau} ß$ einschließlich der auch in Abb. 1 am Tetramethylzinn beobachteten Satelliten ist übriggeblieben. Dieses Triplett wird einem analogen Radikal wie dem im Tetramethylzinn gebildeten ${ }^{10}$ zugeordnet:<smiles>C[Sn](C)(Cl)Cl</smiles>

$$
\begin{aligned}
& g=2,009 \\
& a_{\mathrm{H}}=20 \mathrm{G} \\
& a_{\mathrm{Sn}}=160 \mathrm{G}
\end{aligned}
$$

Das Haupttriplett stammt von der Hfs-Wechselwirkung zweier äquivalenter $\alpha$-Protonen, während die Satelliten von der Hfs-Wechselwirkung der magnetischen Zinnisotope herrühren. Im Vergleich zum Tetramethylzinn beträgt die isotrope Zinn-Hfs-Kopplung hier ca. 160 Gauß. Die größere Hfs-Aufspaltung dürfte durch eine vom Chloratom verursachte größere Spindichte am Zinnatom hervorgerufen werden. Auch die stärkere Spin-Bahn-Kopplung, die am größeren $g$-Faktor erkennbar ist $(2,009$ bei Trimethylzinnchlorid; 2,0067 beim Tetramethylzinn) kann auf die Anwesenheit des Chloratoms zurückzuführen sein. Bemerkenswert ist der Befund, daß im Trimethylzinnchlorid (Abb. 3) im Gegensatz zum Tetramethylzinn (Abb. 1) und Hexamethyldistannan (Abb. 3) bei $g=2,02$ keine breite asymmetrische Resonanz von dreibindigen Zinnradikalen gefunden wurde. Sofern solche Radikale der Form $\left(\mathrm{CH}_{3}\right)_{3} \mathrm{Sn}$. oder $\left(\mathrm{CH}_{3}\right)_{2} \mathrm{ClSn} \cdot$ gebildet werden, so müßten sie unter den vorliegenden Bedingungen sehr rasch weiterreagieren.

\section{Hexamethyldistannan}

Das in Abb. 4 gezeigte ESR-Spektrum des bestrahlten Hexamethyldistannan enthält die bereits an anderen bestrahlten Organozinn-Verbindungen beobachtete breite asymmetrische Linie bei $g=2,02$, die wie beim Tetramethylzinn einem dreibindigen Zinnradikal zugeordnet wird. Aus dem Spektrum kann man allerdings nicht entscheiden, ob dabei die Sn-Sn-Bindung aufgebrochen wird, oder ob eine Methylgruppe wie beim Tetramethylzinn abgetrennt wird. Bei $g=2,004$ erscheint im Spektrum eine ein- 


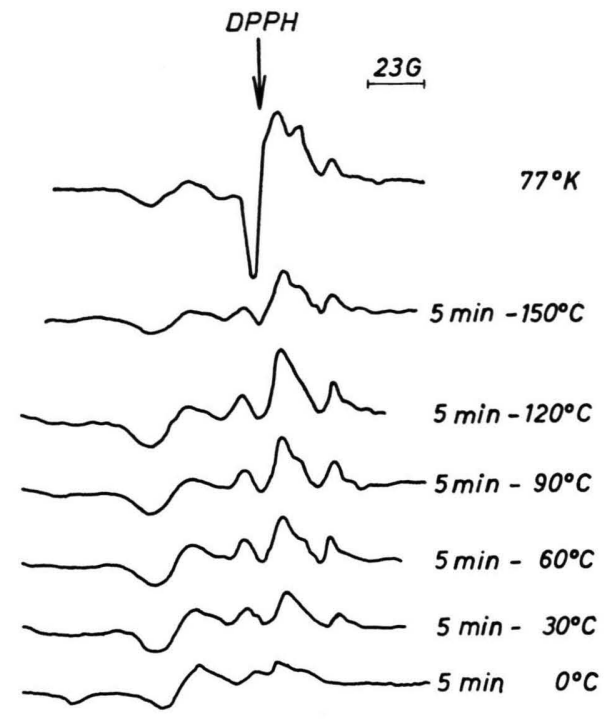

Abb. 4. ESR-Spektrum von $\left(\mathrm{CH}_{3}\right)_{3} \mathrm{Sn}-\mathrm{Sn}\left(\mathrm{CH}_{3}\right)_{3}$ nach Röntgen-Bestrahlung bei $77^{\circ} \mathrm{K}$ und anschließender Erwärmung der Probe (wie in Abb. 1).

zelne etwa $7 \mathrm{Gauß}$ breite Linie, die beim Tempern bereits bei $-150{ }^{\circ} \mathrm{C}$ verschwindet und deren Herkunft ungewiß ist. Übrig bleibt bei $g=2,006$ ein schwaches $1: 2: 1$-Triplett mit einer Aufspaltung von $21 \pm 3 \mathrm{Gau}$, das durch Abtrennung eines Methylgruppen-Wasserstoffs durch Hfs-Wechselwirkung mit zwei $\alpha$-Protonen erklärt werden kann und neben anderen Radikaltypen auf die Existenz eines Radikals vom Typ I hinweist, wie es in Tetramethylzinn und Trimethylzinnchlorid gebildet wird.

\section{Tetraphenylzinn}

Zum Vergleich mit den Ergebnissen an aliphatischen Organozinn-Verbindungen wurde auch das bei Zimmertemperatur feste Tetraphenylzinn bestrahlt und gemessen. Nach Tieftemperatur-Bestrahlung wurde bei $g=2,002$ eine etwa 24 Gauß breite einzelne Linie gefunden, deren Intensität bei gleicher Bestrahlungsdosis um zwei Größenordnungen geringer war als die der untersuchten aliphatischen Organozinn-Verbindungen (Abb. 5).

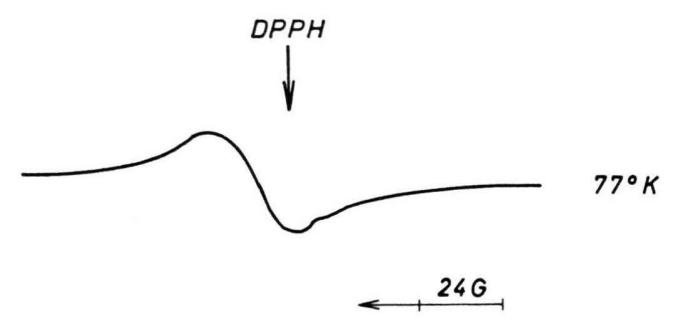

Abb. 5. ESR-Spektrum von Tetraphenylzinn nach RöntgenBestrahlung bei $77^{\circ} \mathrm{K}$.

Die Radikale dieses Spektrentyps, der auch nach Bestrahlung bei Zimmertemperatur entsteht, erwiesen sich als ungewöhnlich stabil. Ein stufenweises Erwärmen der Probe von $77^{\circ} \mathrm{K}$ bis auf $+150{ }^{\circ} \mathrm{C}$ für 1,5 Stunden ergab keinerlei Spektrenveränderungen, sondern lediglich einen geringen Intensitätsverlust. Auch nach Öffnen der Ampulle veränderte der hinzutretende Luftsauerstoff das Spektrum nicht.

Das im Tetraphenylzinn gebildete Radikal muß auf Grund des $g$-Faktors, der typischen Linienbreite von $24 \mathrm{Gau}$ und der großen Stabilität einem mesomeren Radikal mit $\pi$-Elektronensystemen zugeordnet werden. Eine Einbeziehung des Zinnkernes in die Hfs-Wechselwirkung ist wegen der starken Delokalisation des ungepaarten Elektrons und der dadurch bedingten geringeren $\mathrm{Hfs}$-Aufspaltungen im polykristallinen Festkörper nicht zu erkennen. Dieses Radikal zeichnet sich durch ungewöhnlich hohe Stabilität im Wirtsgitter aus. 